\title{
RIP140 in monocytes/macrophages regulates osteoclast differentiation and bone homeostasis
}

\author{
Bomi Lee, ${ }^{1}$ Urszula T. Iwaniec, ${ }^{2}$ Russell T. Turner, ${ }^{2}$ Yi-Wei Lin, ${ }^{1}$ Bart L. Clarke, ${ }^{3}$ Anne Gingery, ${ }^{4}$ \\ and $\mathrm{Li}-\mathrm{Na} \mathrm{Wei}^{1}$ \\ 'Department of Pharmacology, University of Minnesota Medical School, Minneapolis, Minnesota, USA. ${ }^{2}$ Skeletal Biology \\ Laboratory, School of Biological and Population Health Sciences, College of Public Health and Human Sciences, Oregon \\ State University, Corvallis, Oregon, USA. ${ }^{3}$ Division of Endocrinology, Diabetes, Metabolism, and Nutrition, Mayo Clinic, \\ Rochester, Minnesota, USA. ${ }^{4}$ Division of Orthopedic Research, Mayo Clinic, Rochester, Minnesota, USA.
}

\begin{abstract}
Osteolytic bone diseases, such as osteoporosis, are characterized by diminished bone quality and increased fracture risk. The therapeutic challenge remains to maintain bone homeostasis with a balance between osteoclast-mediated resorption and osteoblast-mediated formation. Osteoclasts are formed by the fusion of monocyte/macrophage-derived precursors. Here we report, to our knowledge for the first time, that receptor-interacting protein 140 (RIP140) expression in osteoclast precursors and its protein regulation are crucial for osteoclast differentiation, activity, and coupled bone formation. In mice, monocyte/macrophage-specific knockdown of RIP140 (m\$RIP140KD) resulted in a cancellous osteopenic phenotype with significantly increased bone resorption and reduced bone formation. Osteoclast precursors isolated from $\mathrm{m} \phi \mathrm{RIP140KD}$ mice had significantly increased differentiation potential. Furthermore, conditioned media from m $\phi R I P 140 K D$ primary osteoclast cultures significantly suppressed osteoblast differentiation. This suppressive activity was effectively and rapidly terminated by specific Syk-stimulated RIP140 protein degradation. Mechanistic analysis revealed that RIP140 functions primarily by inhibiting osteoclast differentiation through forming a transcription-suppressor complex with testicular receptor 4 (TR4) to repress osteoclastogenic genes. These data reveal that monocyte/macrophage RIP140/ TR4 complexes may serve as a critical transcription regulatory complex maintaining homeostasis of osteoclast differentiation, activity, and coupling with osteoblast formation. Accordingly, we propose a potentially novel therapeutic strategy, specifically targeting osteoclast precursor RIP140 protein in osteolytic bone diseases.
\end{abstract}

Conflict of interest: The authors have declared that no conflict of interest exists.

Submitted: September 6, 2016 Accepted: February 16, 2017 Published: April 6, 2017

\section{Reference information:} JCI Insight. 2017;2(7):e90517. https:// doi.org/10.1172/jci.insight.90517.

\section{Introduction}

Osteoporosis is a major health concern and is characterized by loss of bone mineral density and bone quality. Osteoporosis has been reported to affect an estimated 10 million Americans over 50 years old and results in 1.5 million fragility fractures each year (1). Bone homeostasis requires a balance between osteoclast-mediated bone resorption and osteoblast-mediated bone formation $(2,3)$. Pathological conditions such as osteoporosis, rheumatoid arthritis, and osteolytic bone metastases result as a consequence of defective coupling of bone formation to the prevailing level of bone resorption; uncoupled bone turnover is responsible, at least in part, for bone loss, increased fracture risk, and deterioration in bone microarchitecture (4). Current therapeutic strategies are primarily antiresorptive; however, these therapies have limitations, as bone formation is further reduced due to the attenuation of osteoclast activity (5). Bone turnover is a dynamic process in which bone is first resorbed by osteoclasts. Osteoblasts then follow and form bone. This process is tightly coupled in healthy bone. Therefore, therapeutics that promote active, coupled, and balanced bone remodeling are needed.

It has become increasingly evident that metabolism has a substantial impact on skeletal health and the coupling of bone formation and resorption $(6,7)$. Metabolic effects on skeletal health present an increasingly recognized medical concern, as obesity, metabolic syndrome, and type 2 diabetes are on the rise (8-10). It was once thought that increased body mass would be protective to the skeleton, but this has now been put 
to question $(11,12)$. As recently reviewed, the beneficial skeletal effects of increased weight can be negated by metabolic dysfunction (13). For instance, women with type 2 diabetes mellitus (T2DM) have a normal or higher bone mineral density; however, they have nearly double the risk of fracture, indicating that overall quality of the bone is reduced (14). We have previously shown that the coregulator, receptor-interacting protein 140 (RIP140), plays an important role in macrophage polarization. RIP140 is a coactivator for $\mathrm{NF}-\kappa \mathrm{B}$ in proinflammatory (M1) macrophages and its degradation mediates resolution of inflammation (15). RIP140 is also a cytosolic repressor of alternative, antiinflammatory (M2) polarized macrophages (16). In mice, lowering macrophage-specific RIP140 expression levels reduces M1 macrophages, increases M2 macrophages, prevents high-fat diet-induced insulin resistance, and enhances insulin sensitivity and white adipose tissue browning even under a high-fat diet (17).

Osteoclasts are derived from the fusion of monocytes/macrophages (18). Given the prominent activities of RIP140 in the monocyte/macrophage lineage, we speculated a potential function of RIP140 in osteoclastogenesis. Macrophage colony-stimulating factor (M-CSF) activation of the M-CSF receptor/ colony stimulating factor (c-Fms) and receptor activator of NF-kB ligand (RANKL) activation of its receptor RANK are important signaling events that trigger osteoclast precursor proliferation and differentiation $(19,20)$. RANKL signaling activates transcription factors, such as nuclear factor of activated $\mathrm{T}$ cells cytoplasmic 1 (NFATc1) and c-Fos, to upregulate osteoclast functional genes such as tartrate-resistant acid phosphatase (TRAP, Acp5) and cathepsin K (Ctsk) (21).

Our in vivo data indicate that RIP140 expression in osteoclasts plays a role in osteoclast differentiation, activity, and bone turnover. Here we present evidence for a physiological role of RIP140 in osteoclast precursors by regulating osteoclast differentiation through the formation of a suppressive transcription regulatory complex with testicular receptor 4 (TR4). We also show that degradation of RIP140 is key to osteoclastogenesis and bone homeostasis, and suggest a potential novel therapeutic strategy by targeting RIP140 protein in the treatment of osteolytic bone diseases.

\section{Results}

Loss of osteoclast precursor RIP140 in vivo results in decreased cancellous volume. Given RIP140's early expression and biological activity in the monocyte-macrophage lineage, and that osteoclasts differentiate from monocytes/macrophages, we speculated a functional role for RIP140 in osteoclast biology. To determine the functional relevance of RIP140 in osteoclast-associated bone homeostasis in animals, we carefully examined the bone phenotype of a monocyte/macrophage-specific RIP140-knockdown transgenic mouse model, m $\phi$ RIP140KD. As described in our previous study (15), endogenous microRNA was used to mimic RIP140-specific shRNA driven by the human CD68 promoter in $\$ \phi R I P 140 \mathrm{KD}$ mice. In vitro osteoclast differentiation was first carried out using bone-marrow cells from tibias and femurs of WT and m $\phi$ RIP140KD mice, and cells were induced with RANKL and M-CSF for 2 days. The expression of osteoclast marker genes Nfatc1, Ctsk, and Acp5 in m $\phi$ RIP140KD cultures was significantly elevated following RANKL treatment as compared with WT (Supplemental Figure 1A; supplemental material available online with this article; https://doi.org/10.1172/jci.insight.90517DS1), suggesting a suppressive role for RIP140 in osteoclastogenesis. RIP140 KD efficiency was confirmed (Supplemental Figure 1A).

To further validate $\mathrm{m} \phi \mathrm{RIP} 140 \mathrm{KD}$ in suppressing osteoclastogenesis in vivo, we performed micro-computed tomography $(\mu \mathrm{CT})$ and histomorphometric analysis of WT and $\mathrm{m} \phi \mathrm{RIP} 140 \mathrm{KD}$ bones. A representative $\mu \mathrm{CT}$ image (Figure 1A) of the femoral diaphysis and metaphysis shows cortical and cancellous bone volume fraction in control and $\mathrm{m} \phi \mathrm{RIP} 140 \mathrm{KD}$ mice. Significant differences in cortical bone parameters in the femur diaphysis were not detected with genotype (Figure 1B). Additionally, reference point indentation material properties assessed ex vivo on the tibia of control and $m \phi R I P 140 \mathrm{KD}$ mice were not significantly different (Supplemental Figure 1B). In contrast, bone volume fraction (calculated as percentage bone volume/tissue volume $[\mathrm{BV} / \mathrm{TV}])$ at both the distal femur metaphysis (47\%) and the fifth lumbar vertebra (L5, 18\%) was significantly lower in m $\phi$ RIP140KD mice compared with WT mice (Figure 1, C and D). Significant reductions in cancellous bone volume fraction in the femur metaphysis were associated with a significant reduction in connectivity density and trabecular number and thickness (Figure 1C). There were no significant differences in trabecular spacing, though it approached significance $(P=0.059)$ (Figure $1 C)$. For L5, the bone volume fraction and trabecular thickness were also significantly reduced and trabecular spacing was significantly increased in vertebrae in m $\phi$ RIP140 mice (Figure 1D). These $\mu \mathrm{CT}$ and histomorphometric analyses revealed that moRIP140KD mice have an osteopenic phenotype with decreased cancellous bone volume. 
A Femur

WT

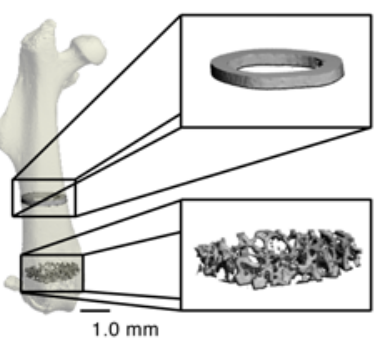

$1.0 \mathrm{~mm}$
RIP140

KD

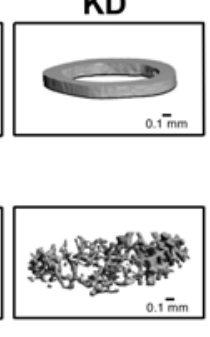

B Femur

Diaphysis

Metaphysis
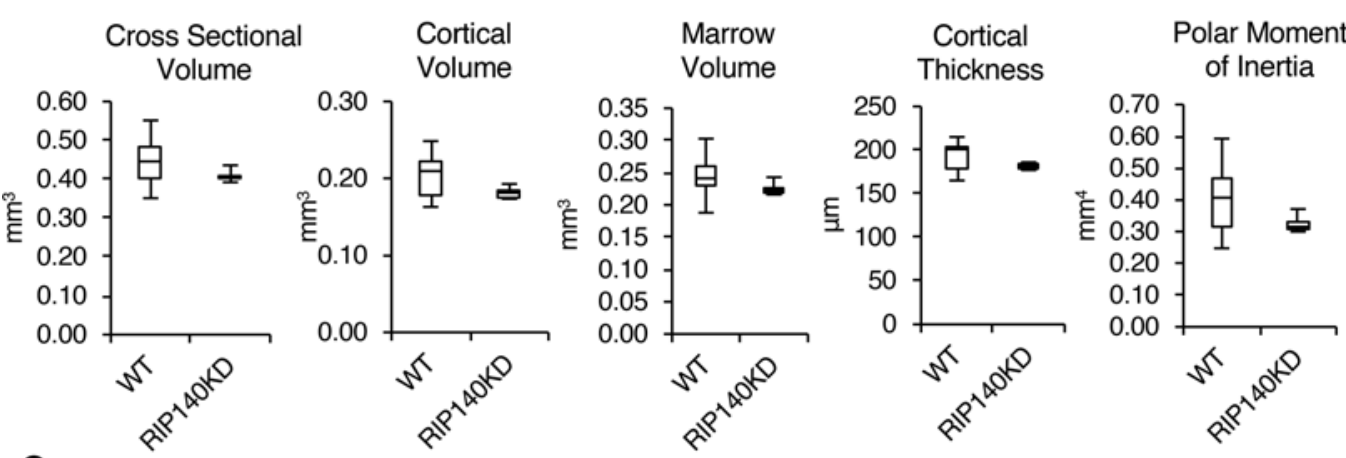

C Femur

Bone Volume

Connectivity
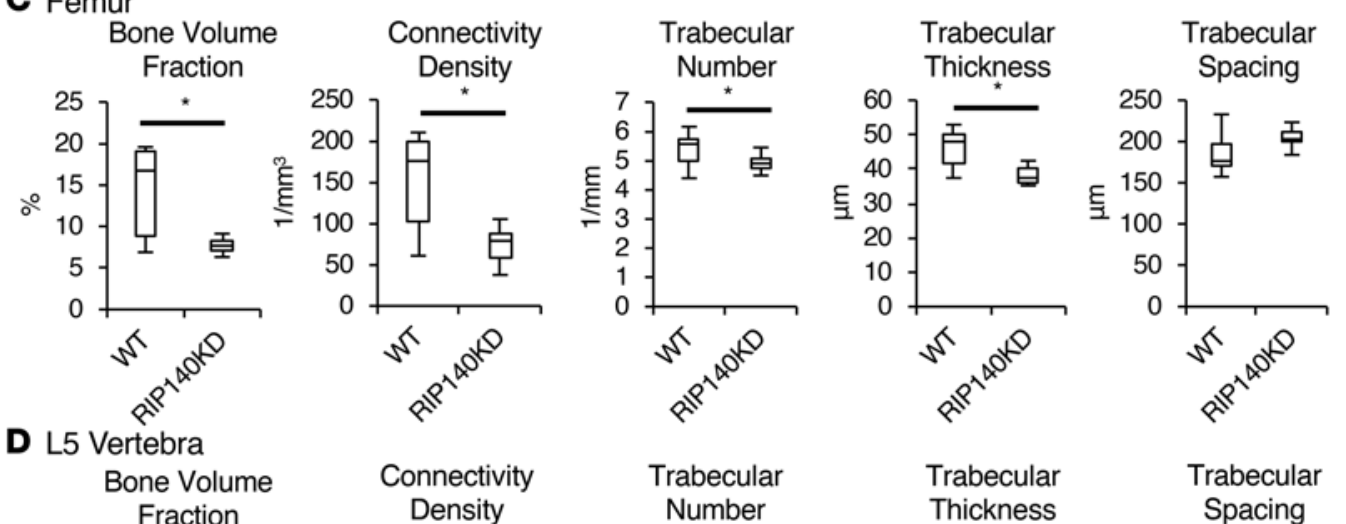
Trabecular Number
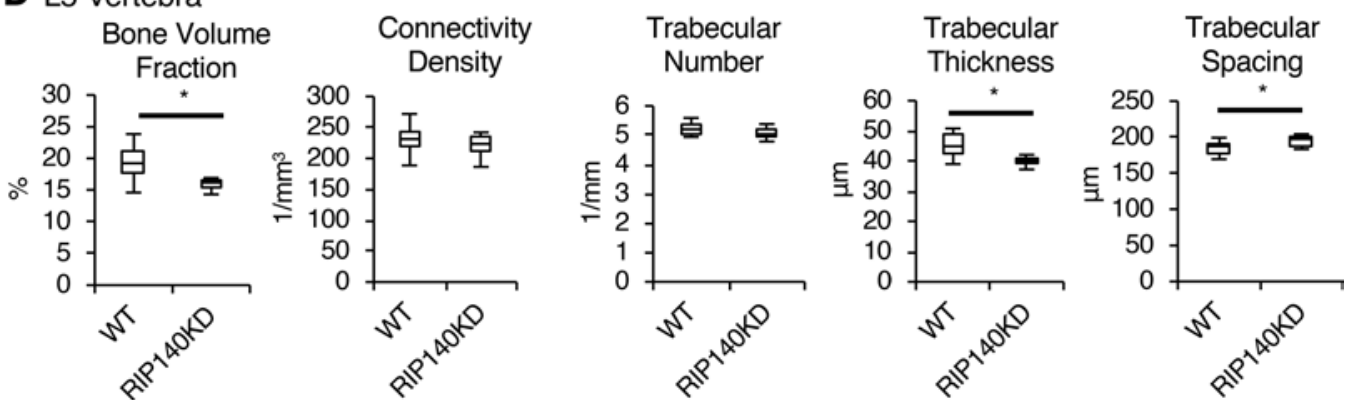

Figure 1. Femoral and vertebral trabecular bone is significantly decreased in mice with reduced RIP140 expression. (A) Representative $\mu \mathrm{CT}$ images of the femoral diaphysis and metaphysis illustrating differences in cancellous bone volume fraction in 9-week-old male WT and monocyte/macrophage-specific RIP140-knockdown (m\$RIP140KD) mice. (B) $\mu \mathrm{CT}$ analysis of cortical bone in the femur diaphysis: cross-sectional volume, cortical volume, marrow volume, cortical thickness, and polar moment of inertia. (C) $\mu \mathrm{CT}$ analysis of cancellous bone in the distal femur metaphysis: bone volume fraction; connectivity density; trabecular number, thickness, and spacing. (D) $\mu \mathrm{CT}$ analysis of cancellous bone in 5th lumbar (L5) vertebra: bone volume fraction; connectivity density; trabecular number, thickness, and spacing. Data are presented as means $\pm \mathrm{SD}$. *Denotes significance at $P<0.05, n=8-10 /$ group.

Loss of $m \phi R I P 140$ in osteoclast precursors results in increased osteoclast activity, reduced osteoblast activity, and reduced bone formation. Bone turnover involves coupling of osteoblast-mediated bone formation to osteoclast-mediated bone resorption. To examine the effect of reducing RIP140 on the osteoclast-osteoblast coupling, we assessed bone resorption and formation indices in $\mathrm{m} \phi \mathrm{RIP} 140 \mathrm{KD}$ mice. The effects of geno- 
A
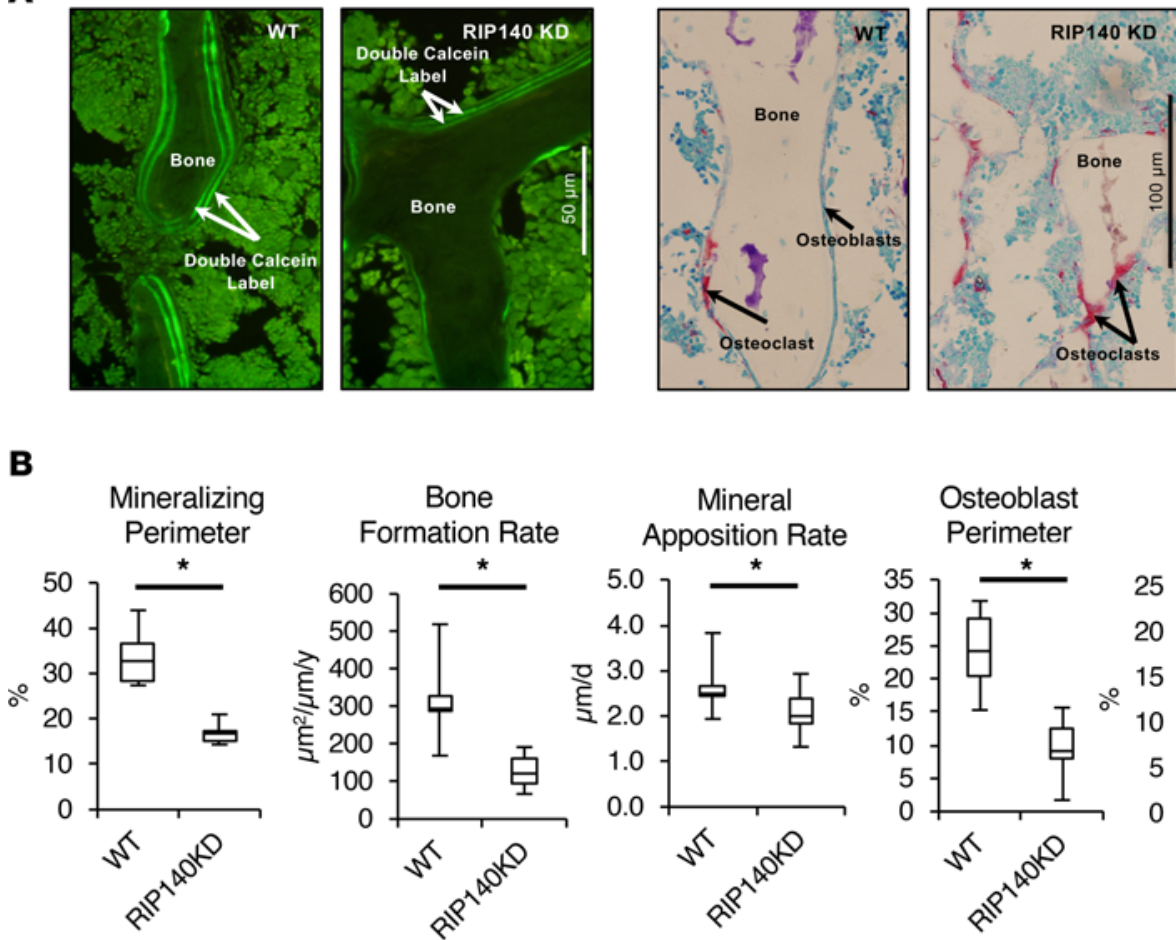

\section{Osteoclast}

Perimeter

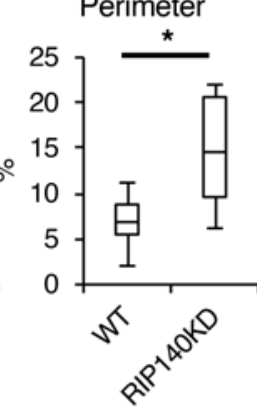

C
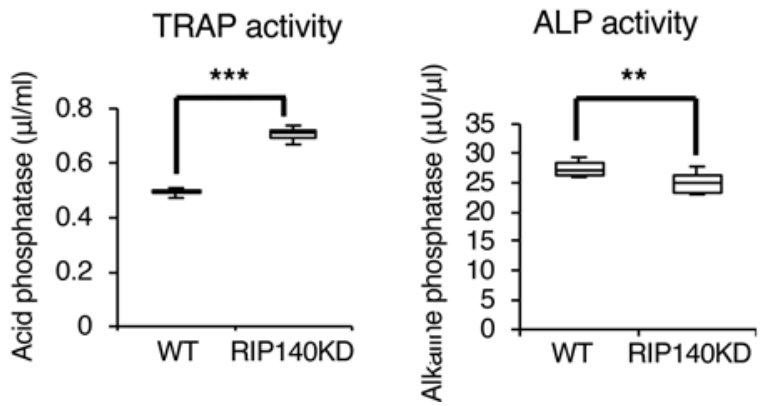

Figure 2. Loss of RIP140 results in increased osteoclast activity, reduced osteoblast activity, and reduced bone formation. (A) Photomicrographs illustrating differences in fluorochrome label incorporation (left) and osteoclast/ osteoblast perimeters (right) in 9-week-old-male WT and monocyte/macrophage-specific RIP140-knockdown ( $\mathrm{m} \phi \mathrm{RIP140KD}$ ) mice. Scale bar: $50 \mu \mathrm{m}$. (B) Indices of bone formation (mineralizing perimeter, bone formation rate, mineral apposition rate, osteoblast perimeter), and bone resorption (osteoclast perimeter) in control and m $\phi R I P 140 K D$ mice at 9 weeks of age. Data are presented as mean \pm SD. ${ }^{*}$ Denotes significance at $P<0.05, n=9-10 /$ group. (C) Tartrate-resistant acid phosphatase (TRAP) activity in bone marrow-derived osteoclasts from WT and m $\phi$ RIP140KD mice ( $n=3$ mice/group) after RANKL and M-CSF treatment for 5 days (left). Alkaline phosphatase (ALP) activity in serum samples from WT and m $\phi R I P 140 K D$ mice ( $n=9$ /group). Data are representative of 3 experimental repeats (mean \pm SD). ${ }^{*} P<0.05,{ }^{* *} P<0.01,{ }^{* *} P<0.001$ by Student's $t$ test.

type on bone formation (incorporation of label into mineralizing bone) and bone resorption (osteoclast perimeter) can be appreciated in Figure 2A. Mineralizing perimeter, bone formation rate, and mineral apposition rate were significantly reduced $(-50 \%,-59 \%$, and $-19 \%$, respectively) in the femur metaphysis of m\$RIP140KD mice as compared with WT (Figure 2B). Osteoblast perimeter/bone perimeter was significantly decreased $(-60 \%)$ with a concomitant increase $(+51 \%)$ in osteoclast perimeter/bone perimeter in m $\phi$ RIP140KD mice, indicating uncoupling of osteoclast and osteoblast formation (Figure 2B). Furthermore, TRAP activity, a mature osteoclast functional indicator, was increased, whereas osteoblast alkaline phosphatase was significantly reduced (Figure $2 \mathrm{C}$ ), further confirming that the coupling has been affected by reducing RIP140 expression to enhance osteoclastogenesis.

To further confirm the effect of RIP140 KD in osteoclast differentiation in vitro, we employed osteoclast differentiation assay with bone marrow cells from WT and m $\phi$ RIP140KD mice (22). After 5 days incubation 
A

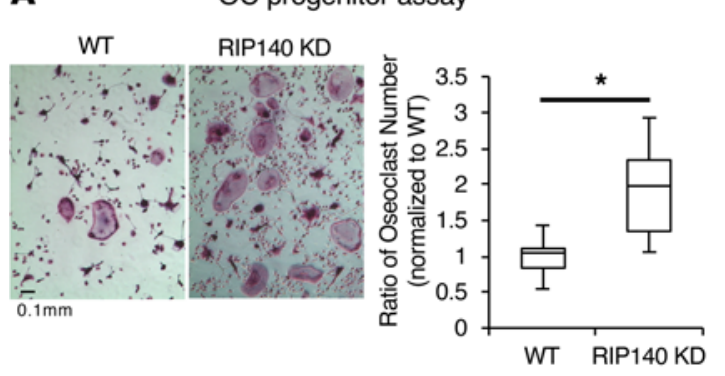

Figure 3. Loss of RIP140 in osteoclasts results in increased osteoclast differentiation and reduced osteoblast markers. (A) Representative images and results of tartrate-resistant acid phosphatase (TRAP)-stained osteoclast (OC) differentiated from bone marrow from WT and monocyte/macrophage-specific RIP140knockdown (m\$RIP140KD) mice $(n=3)$ after 5 days of differentiation with RANKL and M-CSF treatment. Cell quantification data were normalized to WT values and are representative of 3 experimental repeats (mean $\pm S D$ ), $n=3-4$. ${ }^{*} P<0.05$ by Student's $t$ test. Scale bar: $0.1 \mathrm{~mm}$. (B) qRT-PCR analyses of osteoblast markers in primary osteoblasts incubated with conditioned medium of osteoclasts differentiated (5 days) from bone marrow cells in WT and m $\phi R I P 140 K D$ ( $n=4$ mice/group). Primary osteoblasts derived from mouse long bone (femur and tibia) were incubated with conditioned medium from osteoclasts cultured with or without $50 \mu \mathrm{g} / \mathrm{ml}$ ascorbic acid (AA) treatment for 3 days. qRTPCR data are representative of 3 experimental repeats (mean $\pm \mathrm{SD}$ ). ${ }^{*} P<0.05,{ }^{* *} P<0.01,{ }^{* * *} P$ $<0.001$ by Student's $t$ test. Additional statistical significance shown in $\mathbf{B}$ was determined by 2-way ANOVA.

OC conditioned medium $\rightarrow$ Primary Osteoblasts from long bone

with M-CSF and RANKL, osteoclasts were significantly increased in cultures from $m \phi R I P 140 \mathrm{KD}$ mice (Figure $3 \mathrm{~A}$ ), indicating an increase in osteoclast differentiation when RIP140 levels are low.

Conditioned medium (CM) from osteoclast cultures derived from WT or m $\phi$ RIP140KD bone marrow was used in osteoblasts derived from long bones (Figure 3B) and MC3T3-E1 osteoblasts (Supplemental Figure 2A) to assess in vitro coupling of osteoclasts to bone formation responses. Markers of osteoblast differentiation Runx2, Sp7 (osterix), and Alpl (alkaline phosphatase) were assessed by quantitative reverse transcription PCR (qRT-PCR). Consistent with the result of osteoblast activity in mouse serum, osteoblast marker gene expression was substantially diminished by treatment with $\mathrm{CM}$ of osteoclasts derived from $m \phi R I P 140 K D$ mice (Figure 3B and Supplemental Figure 2A). Additionally, gene expression analysis of osteoclast cultures showed significant regulation of multiple known osteoclast coupling factors (Supplemental Figure 2B) including a significant reduction of the recently identified osteoclast coupling factor Wnt1 (22). These data further support the functional consequence of depleting RIP140 in osteoclasts in vivo; depleting RIP140 enhances osteoclast differentiation and activity, and decreases in vivo osteoblast differentiation, indicating an uncoupling of osteoclasts and osteoblasts.

TR4/RIP140 complex formation and repression of osteoclast gene expression. To examine the underlying mechanism of $m \phi R I P 140$ 's suppressive activity in osteoclast differentiation and activity, we first sought to identify the RIP140 complex involved in early phases of osteoclast differentiation by examining RIP140-containing complexes in the murine preosteoclastic cell line RAW264.7 (23). We introduced a FLAG-RIP140 vector into RAW264.7 cells and immunoprecipitated nuclear FLAG-RIP140 complexes. Mass spectrometry data revealed TR4 $(\mathrm{Nr} 2 \mathrm{c} 2)$, a previously reported nuclear receptor that acts primarily to repress genes $(24,25)$, with the greatest number of unique reads (Figure 4A). TR4/RIP140 complex formation was confirmed by coimmunoprecipitation assays in RAW264.7 cells (Figure 4B) and in 293T cells (Figure 4C). Interestingly, TR4 mRNA and protein expression was downregulated following RANKL stimulation (Figure 4D).

We then performed TR4 KD in RAW264.7 cells under RANKL stimulation and found elevated expression of Nfatc1, Ctsk, and Acp5, markers of osteoclast differentiation (Figure 4E). In contrast, overexpression of TR4 in RANKL-stimulated RAW264.7 cells rendered significant or nearly significant downregulation of osteoclast genes (Supplemental Figure 3A), which further supports a repressive activity of the TR4 complex in osteoclast differentiation (see below for the corepressive activity of RIP140). We validated TR4 binding to endogenous osteoclast marker gene promoters. This binding was reduced by RANKL treatment, 
which was recovered by replenishing RIP140 with doxycycline-induced RIP140 overexpression (Figure 4F). Taken together, these data reveal that RIP140 forms a complex with TR4 in preosteoclastic cells to suppress osteoclast differentiation by directly targeting promoter regions of genes critical for osteoclast differentiation or activity. As cells are stimulated with RANKL for osteoclast differentiation, the protein level of the TR4/RIP140 complex is downregulated, allowing differentiation to proceed.

RIP140's repressive activity and protein degradation in RANKL-stimulated osteoclast differentiation. To further confirm the repressive role of RIP140 in osteoclast differentiation, we performed a gain-of-function study in RANKL-stimulated RAW264.7 cells. Elevating RIP140 levels dampened the expression of differentiation markers (Figure 5A), confirming the repressive activity of RIP140 in RANKL-induced osteoclast differentiation. Interestingly, RANKL stimulation decreased RIP140 protein levels without altering RIP140 mRNA expression (Figure 5, B and C), suggesting posttranslational regulation to lower RIP140 protein levels by RANKL stimulation. We previously identified Syk, a nonreceptor tyrosine kinase that stimulated a RIP140 protein ubiquitination/degradation pathway in M1-polarized macrophages (15). Interestingly, Syk inhibition reduced RANKL-induced RIP140 protein degradation and osteoclast gene expression (Figure 5, B and D). Syk has been previously noted to be an important kinase involved in mature osteoclast cytoskeletal organization (26). Our data imply that Syk may have an additional role: to stimulate RIP140 degradation during osteoclast differentiation, leading to enhanced osteoclast differentiation. To provide further evidence for the significance of RIP140 degradation in this process, lentiviruses carrying either WT or a nondegradable RIP140 mutant (Y3F) were introduced into bone marrow-derived mononuclear cells, which were then treated with M-CSF and RANKL for osteoclast differentiation. As predicted, differentiation markers were dampened by expressing WT RIP140, and completely blocked by expressing Y3F that could not be degraded (Figure 5E). Thus, RIP140 exerts a repressive activity for RANKL-stimulated osteoclast differentiation, and is degraded over time by RANKL treatment, allowing differentiation to proceed. Additionally, we conclude that the TR4/RIP140 complex is a suppressor of osteoclast differentiation genes. RANKL signal triggers RIP140 protein degradation and TR4 mRNA downregulation, providing a physiological break to terminate the potent suppressive activity of RIP140 in osteoclast differentiation.

RIP140 suppresses RANKL signaling. RANKL signaling is known to activate NF- $\mathrm{BB}$ and MAPK to induce osteoclast differentiation (27-29). To determine if RIP140 regulates endogenous signaling components, we overexpressed RIP140 in RAW264.7 cells overnight, induced cells with RANKL, and monitored the activation of endogenous signaling components following RANKL treatment. As shown in Figure 6A, RANKL induced MEK1/2, ERK1/2, and p65 phosphorylation within 30 minutes in control cells, which was reduced by overexpressing RIP140 in RAW264.7 cells, indicating that RIP140 interfered with activation of endogenous signaling. Furthermore, we noted that RANKL-induced TRAP was dose-dependently reduced by overexpressing RIP140 (Figure 6B). Since RIP140 can regulate gene expression by interacting with transcription regulators, we explored whether RIP140 could also act as a transcription coregulator of upstream signaling molecules RANK or TNF receptor-associated factor (TRAF). In chromatin immunoprecipitation (ChIP) assay, RIP140 was not detected on RANK or TRAF promoters, ruling out RIP140 being directly involved in RANKL-dependent NF- $\mathrm{BB}$ and MAPK activation at the transcription level. However, RIP140 was detected on the $N f a t c 1$ promoter (Figure 6C), which was reduced upon RANKL stimulation. TR4 binding to Nfatc1 was significantly enhanced by overexpressing RIP140 in unstimulated cells. In the later (24 hours) phase of RANKL stimulation, TR4 was no longer detected on this gene (Figure 6C). In unstimulated cells, the Nfatc1 promoter was bound by the major activating transcription factors, NFATc1 itself and PU.1, which was enhanced by RANKL treatment. But overexpressing RIP140 reduced binding of these activating transcription factors (Figure 6C), indicating that RIP140 plays a negative role in transcriptional regulation of the osteoclastogenic program. Consistently, RANKL-triggered transcription activation marks such as $\mathrm{H} 3$ acetylation were dampened and transcription silencing markers such as $\mathrm{H} 3 \mathrm{~K} 9$ trimethylation were enhanced by increasing RIP140 expression (Figure 6C). Together, these data show a negative role for RIP140 in regulation of the osteoclastogenic program.

\section{Discussion}

In this study, we report the m $\phi$ RIP140KD mouse model, which exhibits a cancellous osteopenic phenotype with increased osteoclast-lined and reduced osteoblast-lined bone perimeter, ultimately resulting in decreased bone formation rate and cancellous bone volume, similar to what is seen in some forms of osteoporosis (e.g., glucocorticoid-induced osteoporosis) (30). Significant declines in cancellous bone 
A

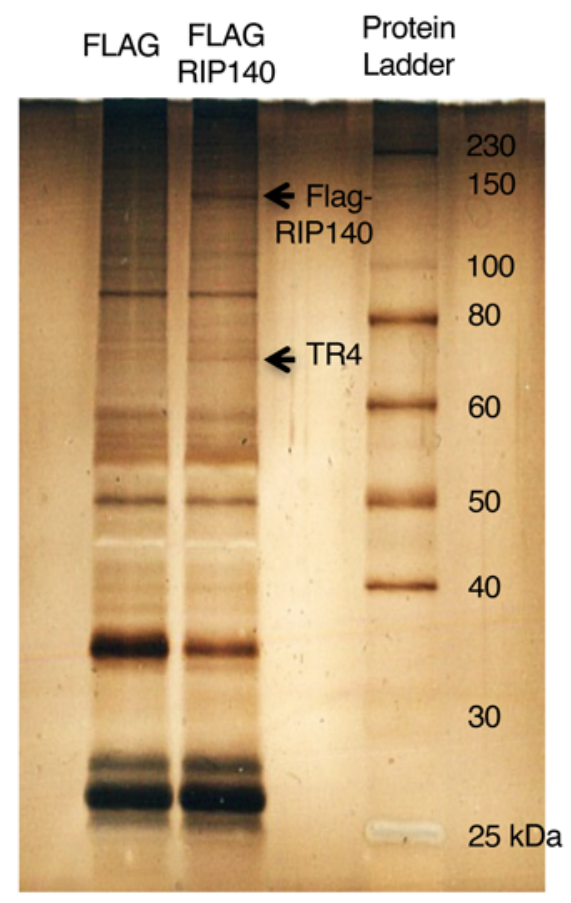

B

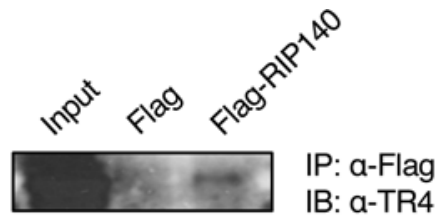

C

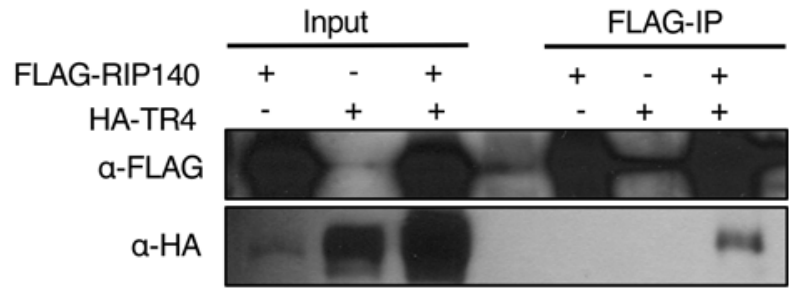

D

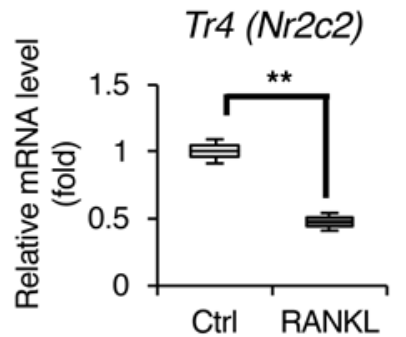

RANKL $(50 \mathrm{ng} / \mathrm{ml}) \quad 0 \quad 1 \quad 2$ (days)

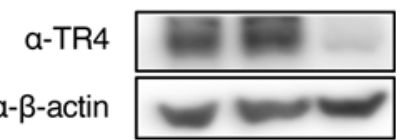

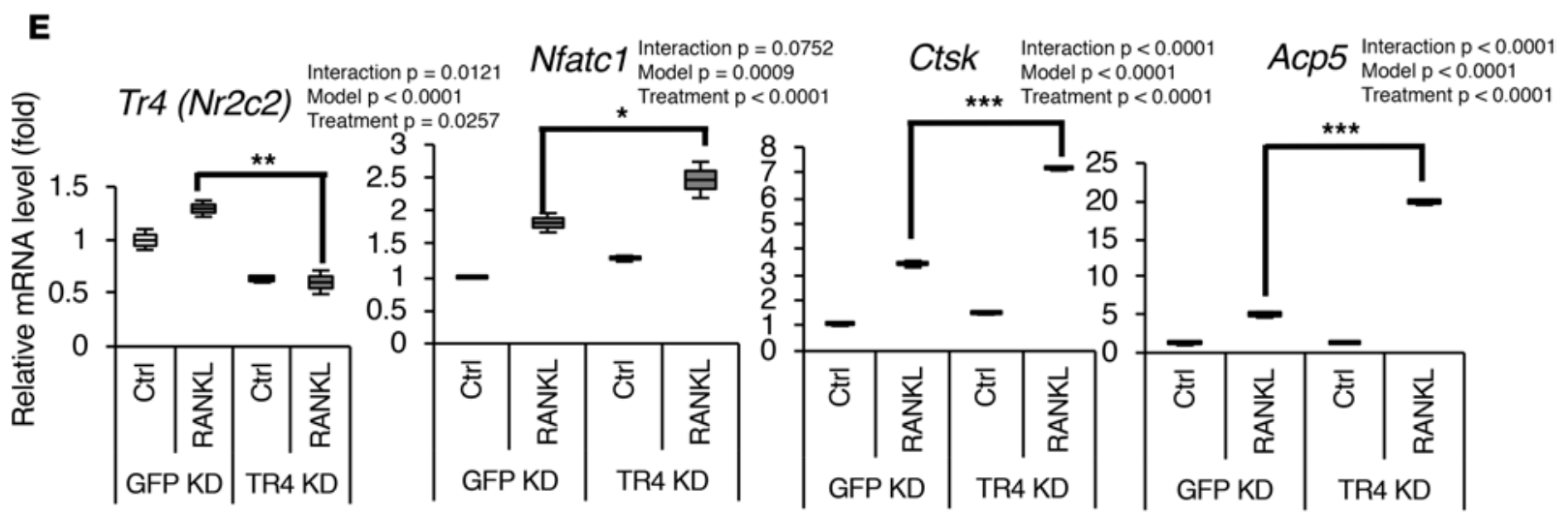

$\mathbf{F}$

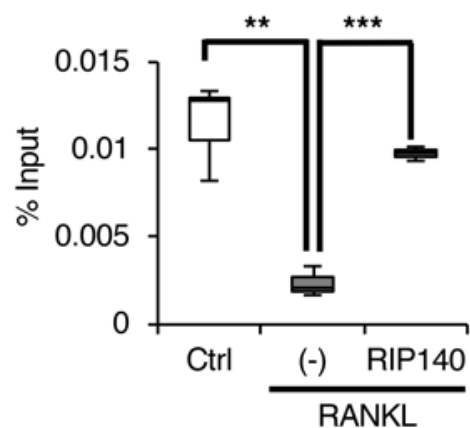

Ctsk promoter

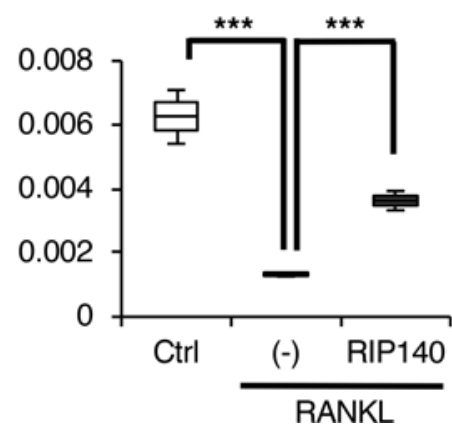

Acp5 promoter

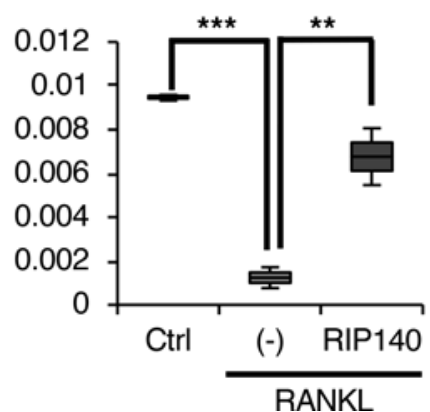

Figure 4. TR4/RIP140 complex formation and repressive activity in osteoclast differentiation. (A) Silver staining of FLAG-RIP140-associated proteins in RAW264.7 cells. (B) Western blot analysis of RIP140/TR4 complex in RAW264.7 cells. (C) Coimmunoprecipitation (IP) of FLAG-RIP140/HA-TR4 from HEK293T. (D) qRT-PCR and Western analyses of TR4 after RANKL stimulation for 2 days. (E) qRT-PCR analysis of osteoclast marker genes in control (GFP KD) versus TR4-knockdown (TR4 KD) RAW264.7 cells with or without RANKL treatment for 24 hours. Additional statistical significance shown in E was determined by 2-way ANOVA. (F) ChIP assay of TR4 on osteoclast marker gene promoters in RAW264.7 cells treated with RANKL stimulation for 30 minutes. RIP140 overexpression was induced by doxycycline pretreatment $(250 \mathrm{ng} / \mathrm{ml})$ for 6 hours. Additional statistical significance was determined by 1-way ANOVA (Nfatc1 promoter $P=0.0014$, Ctsk promoter $P<0.0001$, Acp5 promoter $P<0.0001$ ). Data are representative of 3 experimental repeats (mean $\pm \mathrm{SD})$. For all graphs, Student's $t$ test $(n=3)$ was used unless otherwise specified. ${ }^{*} P<0.05,{ }^{* *} P<0.01,{ }^{* * *} P<0.001$. 
A

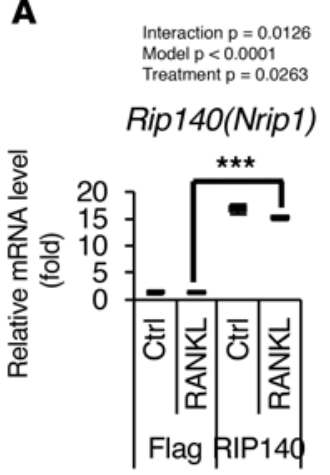

B

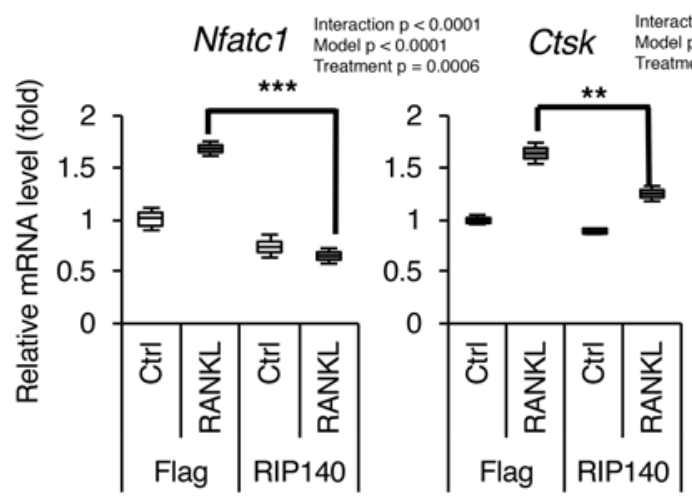

C

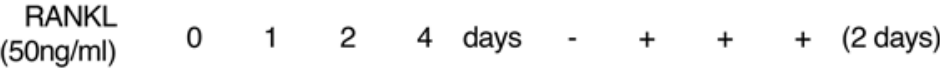

Syk inhibitor

a-RIP140

a- $\beta$-actin

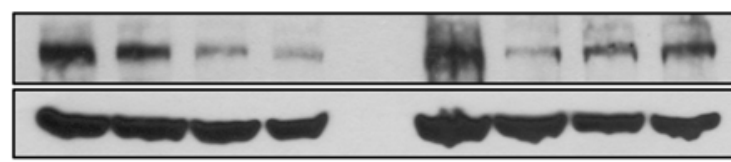

흥

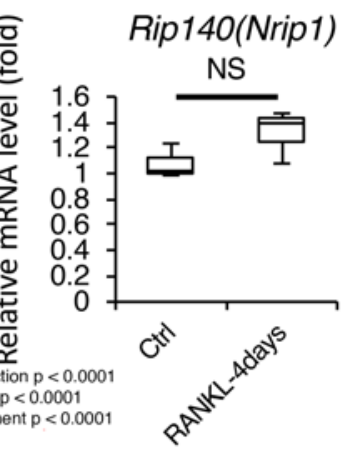

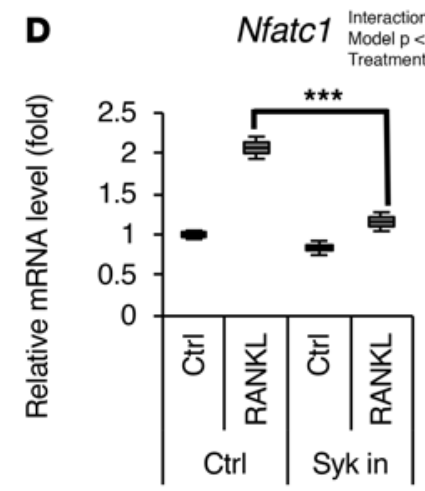

Ctsk Interaction $p<0.0001$ Model $p<0.0001$

$p=0.0002$
0.0001
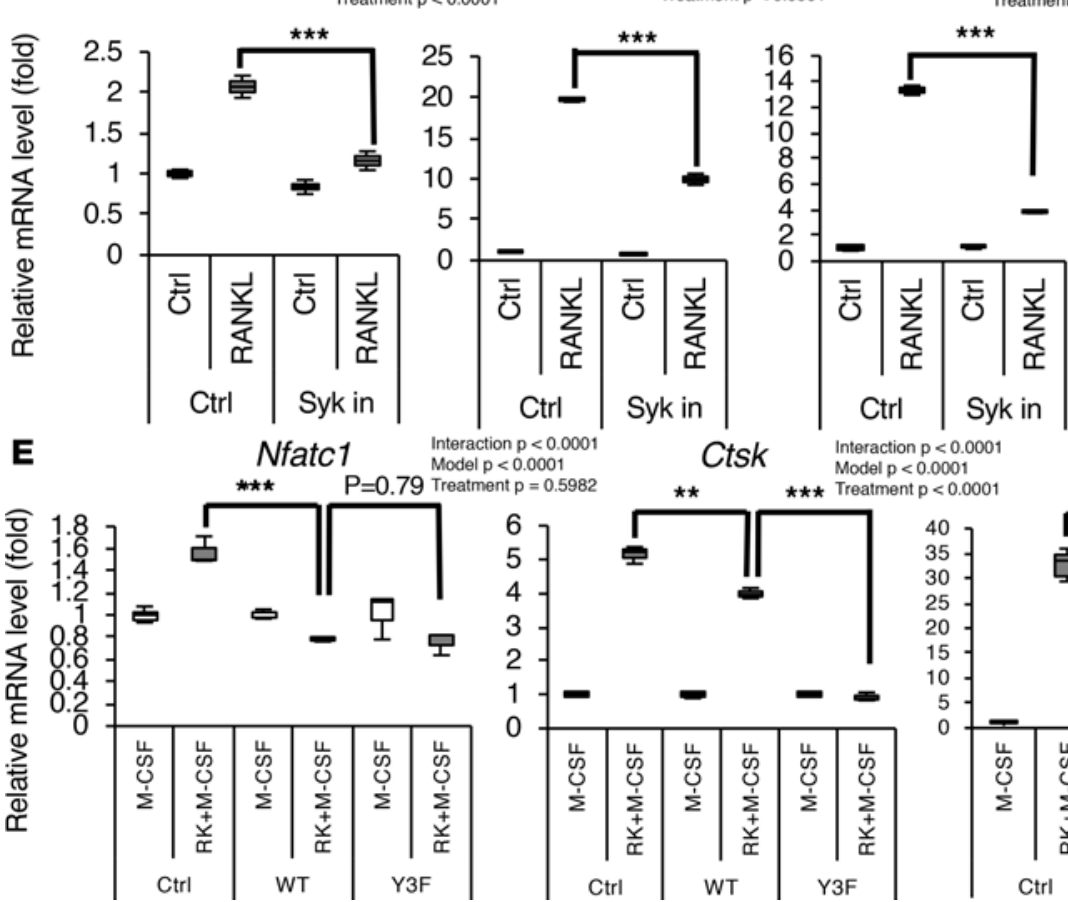

Model $p<0<0.0001$

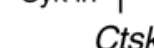

\section{Interaction $p<0.0001$ Model $\mathrm{p}<0.0001$}
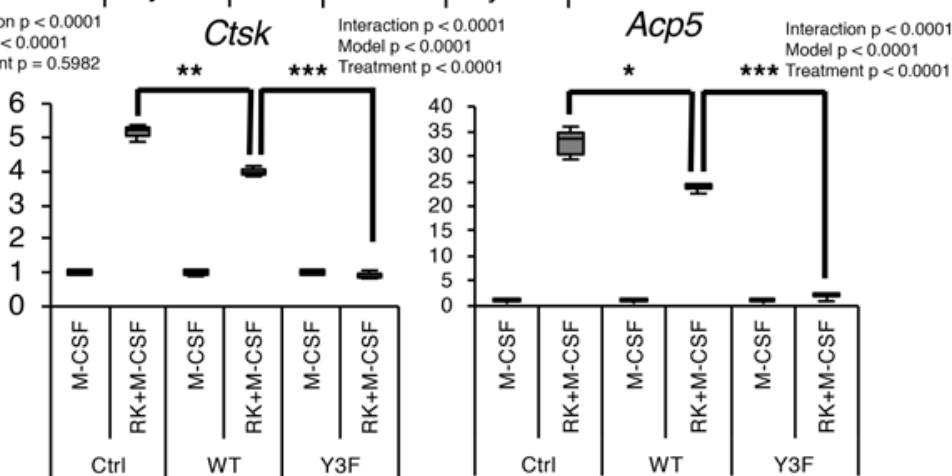

Figure 5. RIP140 represses osteoclast differentiation and is degraded by RANKL treatment in osteoclast differentiation. (A) qRT-PCR analyses of osteoclast markers in control (FLAG) or FLAG-RIP140-overexpressing RAW264.7 cells treated with RANKL for 2 days. (B) Western blot analyses of RIP140 in RAW264.7 cells treated with RANKL for indicated time periods with or without Syk (nonreceptor tyrosine kinase) inhibitor pretreatment. (C) qRT-PCR analyses of RIP140 in RAW264.7 cells with or without RANKL treatment for 4 days. NS, not significant. (D) qRT-PCR analyses of osteoclast markers in RAW264.7 cells treated with RANKL for 2 days with or without Syk inhibitor pretreatment. (E) qRT-PCR analyses. Bone marrow-derived osteoclasts were differentiated with M-CSF and RANKL (RK+M-CSF) for 3 days following transduction with lentiviruses carrying control (Ctrl), WT RIP140, or nondegradable mutant RIP140 (Y3F). Data are representative of 3 experimental repeats (mean \pm SD). For all graphs, Student's $t$ test $(n=3)$ was used unless otherwise specified. ${ }^{*} P<0.05,{ }^{*} P<0.01,{ }^{* *} P<0.001$. Additional statistical significance shown in $\mathbf{A}, \mathbf{D}$, and $\mathbf{E}$ was determined by 2-way ANOVA. 
A

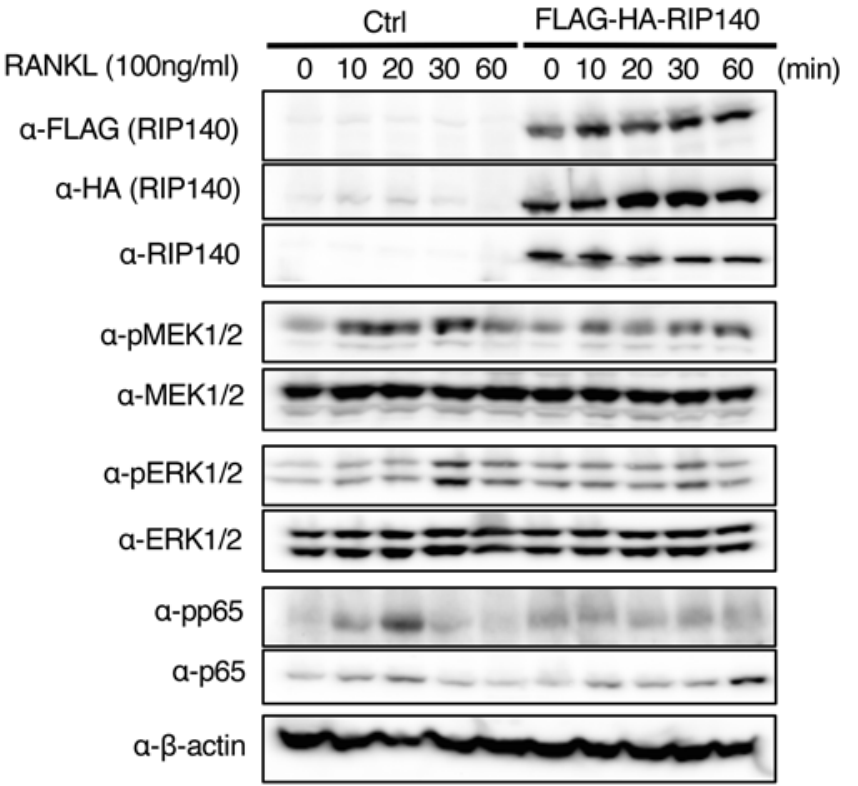

B

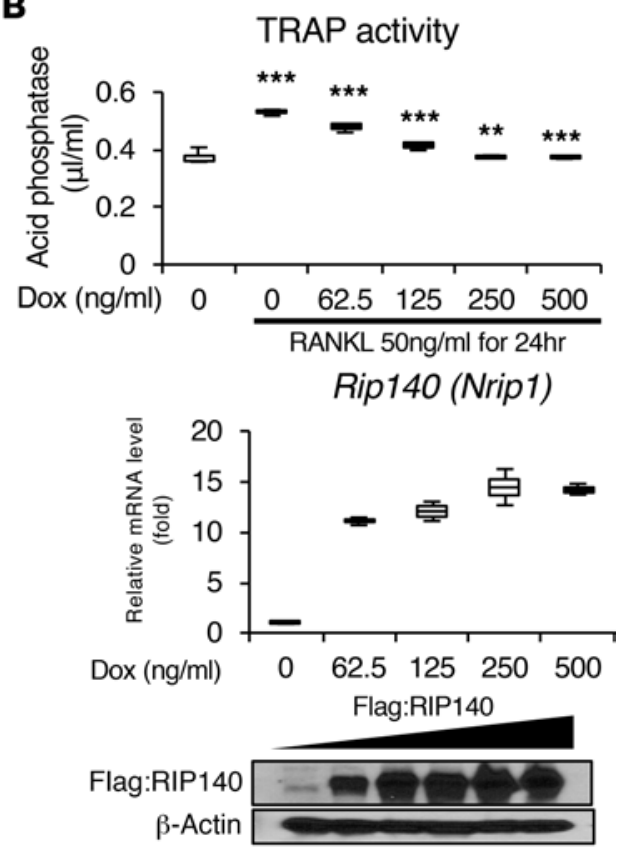

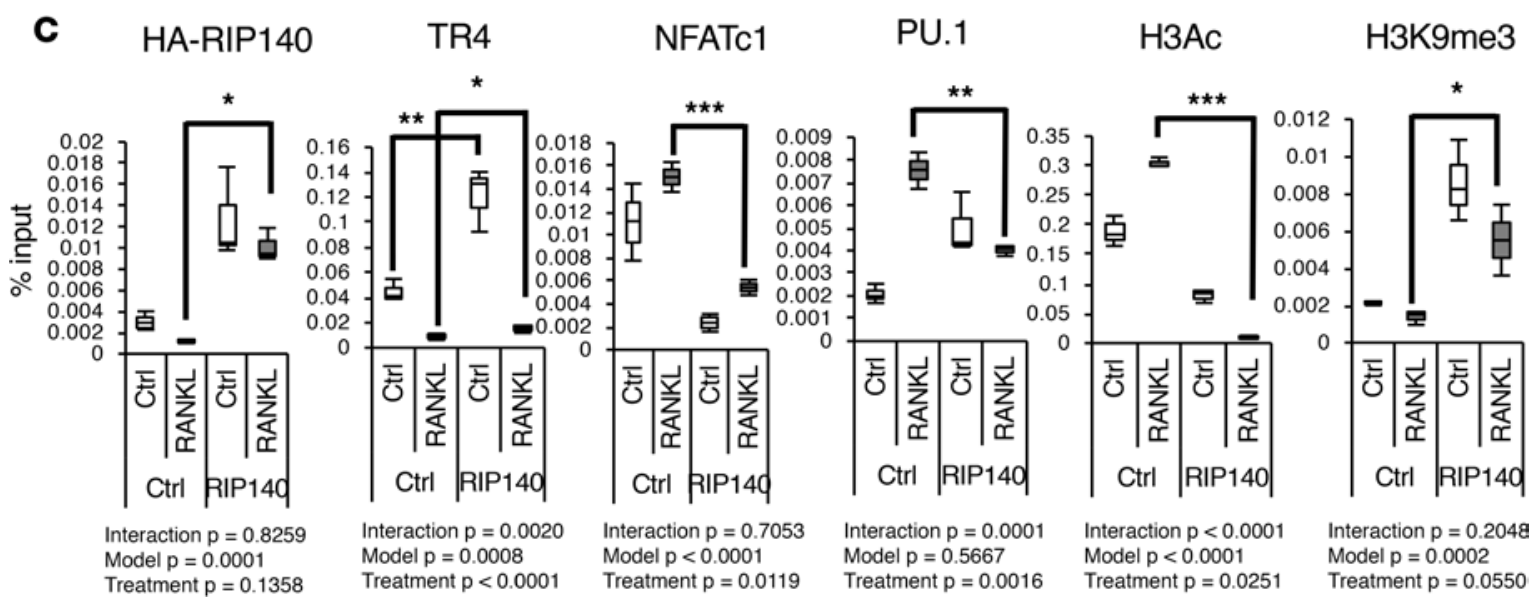

Figure 6. RIP140 suppresses RANKL signaling and transcription activation of osteoclastogenic genes. (A) Western blot analyses of Flag-HA-RIP140 and $\mathrm{MAPK} / \mathrm{NF}-\mathrm{KB}$ signaling components in whole-cell lysates from control versus RIP140-overexpressing RAW264.7 cells. RIP140 overexpression was induced by doxycycline for 16 hours and cells were treated with RANKL at indicated times. (B) Tartrate-resistant acid phosphatase (TRAP) activity in doxycyclineinduced (Dox) RIP140-expressing RAW264.7 cells. Statistical significance was determined with Student's $t$ test $(n=3)$, and each treated sample was compared to unstimulated one. (C) ChIP assay of HA-RIP140, TR4, NFATc1, PU.1, H3Ac, and H3K9me3 on the Nfatc1 promoter in doxycycline-induced RIP140-expressing RAW264.7 cells after RANKL treatment ( 0.5 hours for H3Ac and H3K9me3, 6-24 hours for others). Additional statistical significance in C was determined by 2-way ANOVA. Data are representative of 3 experimental repeats (mean \pm SD). For all graphs, Student's $t$ test ( $n=3$ ) was used unless otherwise specified. ${ }^{*} P<0.05,{ }^{* *} P<0.01,{ }^{* *} P<0.001$.

volume fraction were seen in distal femur metaphysis and lumbar vertebra. The observed deficits in RIP140 KD bone were limited to cancellous compartments, an observation consistent with the proposed mechanism of action. Cancellous bone balance is primarily determined by coupled bone turnover. While cortical bone in humans undergoes lifelong bone remodeling, this is not the case in mice; cortical bone mass in small rodents is primarily determined by longitudinal and radial growth (31). RIP140 in monocyte/macrophage-derived osteoclast precursors acts as a negative regulator of osteoclast differentiation. In WT animals, RANKL treatment attenuates RIP140 action as a result of a reduction in RIP140 protein, and its transcriptional complex partner TR4 mRNA, providing a physiological brake to terminate this critical gatekeeper of osteoclastogenesis. Additionally, the RIP140/TR4 complex targets key genes involved in osteoclast differentiation and activity including Nfatc1, Ctsk, and Acp5. 
Consistent with in vivo studies, primary osteoblasts and osteoblastic MC-3T3 cells cultured with CM from $\mathrm{m} \phi \mathrm{RIP} 140 \mathrm{KD}$ primary osteoclasts had significantly reduced osteoblast marker gene expression. This indicates that RIP140 in osteoclasts is also an important regulator of osteoclast-mediated cross-talk with osteoblasts. Further, transcriptional regulation of osteoclast-derived coupling factors was dysregulated. While some known coupling factors (Sphk1, Pdgfb, Cthrc1, and Bmp2) were not substantially changed, multiple coupling factors were significantly increased, including Bmp6, Wnt10b, and Ctgf in m $\phi$ RIP140KD osteoclasts compared with WT. This may be a compensatory response to the uncoupling between osteoclasts and osteoblasts. Interestingly, we found that Wnt 1 was significantly downregulated in RIP140-ablated osteoclasts. Recent work has shown that Wnt1 is an important osteoclast-derived coupling factor and disruption of this factor results in an uncoupling of osteoclasts-osteoblasts (22).

Here we note that MEK, ERK, and NF- $\mathrm{B}$ signaling was inhibited by RIP140 overexpression, indicating that RIP140 acts, at least in part, to inhibit second messenger activation. Interestingly, and in contrast to our finding in inflammatory macrophages where RIP140 is associated with the NF- $\kappa$ B subunit RelA protein, immunoprecipitation assays with RIP140 and RelA performed here show no interaction in osteoclasts. Further mass spectrometry analysis did not identify RelA as a RIP140KD-interacting protein in preosteoclasts. While RIP140 may regulate second messenger signaling, we also saw that RIP140 is strongly associated with TR4 in osteoclasts as a transcriptional regulatory complex to regulate important osteoclast target gene promoters. This further supports the notion that RIP140 may regulate osteoclast differentiation at both the protein and at the transcriptional level. The anti-osteoclastogenesis potential of the RIP140/TR4 complex is also supported by whole-body TR4 knockout, which leads to postnatal mortality and growth retardation, impaired reproduction and maternal behavior in female mice (32), and impaired skeletal development including osteoporosis with reduced osteoblast activity (33).

Of further significance is the rapid degradation of RIP140 protein upon RANKL treatment mediated by, at least in part, Syk-mediated phosphorylation. This is distinct from the corepressor factor, TR4, whose expression is reduced primarily at the level of mRNA. This suggests a potential new therapeutic strategy for managing bone diseases by blocking osteoclast RIP140 protein degradation to attenuate osteoclastogenesis. It is important to note that previous work on Syk inhibition have found that Syk arrests terminal events of osteoclastogenesis; however, this does not impact the regulation of Nfatc1 at early time points (34). Recent work has shown that Syk activation is important for organization of the mature osteoclast cytoskeleton and subsequent osteoclast activity (26). Additionally, it has been reported that Syk-deficient bone marrow macrophages do not have a delay in differentiation (35). Our findings concerning the protein regulation of RIP140 have uncovered a potentially novel and previously unknown role for Syk in osteoclast differentiation, namely the regulation of RIP140.

Several reports have indicated RIP140 as a candidate gene associated with bone-related traits and osteoporosis in microarray, multilocus single nucleotide polymorphisms (SNPs) analysis (36). Further evidence has shown that RIP140 gene expression in hematopoietic cells is correlated with bone mineral density (37). Additionally, studies of Spanish postmenopausal women detected genetic interactions between RIP140 and other estrogen-related genes such as ESR2 and BMP15 loci, and the involvement of RIP140 in osteoporosis (38). A previous report suggested that a correlation between estrogen signaling in mature osteoclasts may act through RIP140; however, this report provided no direct evidence of an estrogen receptor/RIP140 complex, focused on mature osteoclasts, and used a nonphysiologic model of estrogen withdrawal in both reproductive and skeletally immature animals (39). A recent report also showed cancellous changes in a global knockout model of RIP140; however, given RIP140's known systemic effects, a direct correlation to osteoclast activity is unclear (40). Given RIP140's wide-spectrum activities, such as in metabolism and inflammation, nondiscriminatingly targeting RIP140 might negate the beneficial impact of RIP140 ablation in osteoclast macrophage precursors (15-17).

Importantly, our results have provided unambiguous evidence for RIP140 and the RIP140/TR4 transcription complex playing a role in osteoclastogenesis, and conclude that RIP140 plays a crucial role in maintaining bone health. Increased osteoclast number and bone resorption are characteristic of bone destructive diseases such as osteoporosis, osteolytic bone metastases, and rheumatoid arthritis. Therefore, an important therapeutic strategy for these diseases is to maintain the coupled resorption and formation that is found in healthy bone turnover. Specifically targeted therapeutics that can regulate osteoclast activity and cross-talk with osteoblast activity would be an important leap forward in therapeutic design, rather than relying on substantial inhibition of osteoclast activity or survival. The potential to target osteoclast-specific RIP140 
protein degradation to attenuate osteoclastogenesis and activity is an intriguing strategy. Further studies to investigate the regulation of RIP140 in both signal regulation and transcriptional regulation are warranted.

In summary, our results reveal a potentially novel role for RIP140 in osteoclast differentiation, activity, and bone turnover.

\section{Methods}

Reagents and procedures for cell cultures, mass spectrometry, and immunoprecipitation are described in the supplemental data.

Animals. All studies were carried out using male C57BL/6J mice maintained in the animal facility of the University of Minnesota. Nine-week-old male mice ( $n=8-10 /$ group) were injected with the fluorochrome calcein (12.5 mg/kg; Sigma-Aldrich) 4 days and 1 day prior to sacrifice to label mineralizing bone. $\mathrm{m} \phi \mathrm{RIP} 140 \mathrm{KD}$ mice were generated as described (15). To generate transgenic mice overexpressing shRNA targeting RIP140 in a monocyte/macrophage lineage, endogenous microRNA was used to mimic RIP140specific shRNA (41) and the expression was driven by the human CD68 promoter (42). The DNA fragment was subcloned into the pWhere vector, and the transgenic DNA was excised and injected into C57BL/6 mouse oocytes (Mouse Genetics Laboratory, University of Minnesota). Transgenic founder mice were genotyped by PCR using the following primers: forward, 5'-GAGTTCTCAGACGCTGGAAAGCC-3' and reverse, 5'-GTCCAATTATGTCACACCACAGAAG-3'.

$\mu C T$. $\mu \mathrm{CT}$ was used for nondestructive 3-dimensional evaluation of bone volume and architecture as described (43). Details are provided in supplemental methods.

Histomorphometry. Methods used for measuring static and dynamic bone histomorphometry have been described (44). Details are provided in supplemental methods.

Immunoprecipitation and mass spectrometry. FLAG-RIP140 RAW264.7 cells were treated with or without doxycycline $(250 \mathrm{ng} / \mathrm{ml})$ for 6 hours. Cells were washed with PBS and harvested. Nuclear extracts were prepared using hypotonic buffer, low-salt buffer, and high-salt buffer. Nuclear extracts were treated with Benzonase nuclease (Sigma-Aldrich) and then dialyzed in BC300 (45). After preclearing with mouse IgGagarose beads, nuclear extracts were immunoprecipitated with anti-FLAG M2 magnetic beads (SigmaAldrich) and washed with 0.1\% NP-40-containing BC300. FLAG-RIP140-bound proteins were eluted with FLAG peptides (Sigma-Aldrich), and samples were sent to the Taplin Biological Mass Spectrometry Facility at Harvard Medical School for mass spectrometry analysis. FLAG-RIP140-binding proteins were validated with Western blot analysis using specific antibodies.

Cell culture, TRAP staining, and CM experiments. RAW264.7 (TIB-71) and MC3T3-E1 (CRL-2593) cells were purchased from ATCC. Bone marrow cells were isolated from mouse femurs and tibias by flushing bone marrow with PBS. After lysing red blood cells, bone marrow cells were plated $\left(4 \times 10^{5}\right.$ cells $/$ well $)$ in 24-well plates and incubated with differentiation media ( $\alpha$-MEM medium supplemented with $10 \%$ FBS, $1 \%$ antibiotics, RANKL [100 ng/ml], and M-CSF [25 ng/ml]) for 4 days. On day 5, cells were fixed with $4 \%$ paraformaldehyde and stained for TRAP (Sigma-Aldrich) as previously reported (27). For the CM, FBS-containing fresh $\alpha$-MEM medium was added to primary osteoclasts differentiated with M-CSF and RANKL for 5 days. After a 24-hour incubation, CM was collected and added to MC3T3-E1 cells or primary osteoblasts for 3 days with ascorbic acid $(50 \mu \mathrm{g} / \mathrm{ml})$ for osteoblast differentiation.

DNA constructs, lentivirus production, and transduction. WT-RIP140 and Y3F-RIP140 expression lentivirus constructs were prepared as described (15). The doxycycline-inducible FLAG-HA-RIP140 expression lentivirus construct was made as described (46). Viruses were generated by transfection of lentivirus constructs and packaging plasmids in HEK293T cells. The culture medium including viruses was harvested 2-3 days after transfection, and filtered to remove nonadherent $293 \mathrm{~T}$ cells. Filtered viruses were concentrated with Lenti-X (Clontech, 631232) overnight. Viruses were collected by centrifugation and saved at $-80^{\circ} \mathrm{C}$. For virus transduction in cells, viruses were incubated in Polybrene-containing (EMD Millipore) media for 3 days.

Statistics. In vitro experiments were performed at least 3 times. In vivo experiments $(n=810)$ are reported. Results are presented as box-and-whisker plots, with the center line representing the median. The whiskers indicate the 10th and 90th percentiles. Unpaired and 2-tailed Student's $t$ test was used for both in vitro (parametric) and in vivo (nonparametric) analysis. $P$ values of 0.05 or less were considered statistically significant $\left({ }^{*} P<0.05,{ }^{* *} P<0.01,{ }^{* * *} P<0.001\right)$. Two-way ANOVA was performed to examine the influence of 2 independent factors: the model and the treatment.

Study approval. All the animal studies have been approved by the University of Minnesota IACUC. 


\section{Author contributions}

BL, AG, and LNW designed research. BL and AG performed research.BL, UTI, RTT, YWL, and AG collected data. BL, UTI, RTT, YWL, BLC, AG, and LNW contributed to the discussion, and reviewed and wrote the manuscript.

\section{Acknowledgments}

This work was supported by NIH grants DK54733, DK60521, and DK60521-12S1, the Dean's Commitment and the Distinguished McKnight Professorship of the University of Minnesota (to L.N.W.), and NIH grant F32-AR063596, Mayo Clinic Center for Regenerative Medicine (to A.G.)

Address correspondence to: Li-Na Wei, Department of Pharmacology, University of Minnesota Medical School, Minneapolis, Minnesota 55455, USA. Phone: 612.625.9402; E-mail: weixx009@umn.edu.

1. Offfice of the Surgeon General (US). Bone health and osteoporosis: a report of the Surgeon General. Rockville (MD): Office of the Surgeon General (US); 2004. https://www.ncbi.nlm.nih.gov/books/NBK45513/. Accessed March 6, 2017.

2. Parfitt AM. The coupling of bone formation to bone resorption: a critical analysis of the concept and of its relevance to the pathogenesis of osteoporosis. Metab Bone Dis Relat Res. 1982;4(1):1-6.

3. Martin TJ, Sims NA. Osteoclast-derived activity in the coupling of bone formation to resorption. Trends Mol Med. 2005;11(2):76-81.

4. Cummings SR, et al. Risk factors for hip fracture in white women. Study of Osteoporotic Fractures Research Group. $N$ Engl $J$ Med. 1995;332(12):767-773.

5. Rodan GA, Martin TJ. Therapeutic approaches to bone diseases. Science. 2000;289(5484):1508-1514.

6. Lecka-Czernik B, Rosen CJ. Energy excess, glucose utilization, and skeletal remodeling: New insights. J Bone Miner Res. 2015;30(8):1356-1361.

7. Compston JE, et al. Relationship of weight, height, and body mass index with fracture risk at different sites in postmenopausal women: the Global Longitudinal study of Osteoporosis in Women (GLOW). J Bone Miner Res. 2014;29(2):487-493.

8. Cornier MA, et al. The metabolic syndrome. Endocr Rev. 2008;29(7):777-822.

9. Alberti KG, et al. Harmonizing the metabolic syndrome: a joint interim statement of the International Diabetes Federation Task Force on Epidemiology and Prevention; National Heart, Lung, and Blood Institute; American Heart Association; World Heart Federation; International Atherosclerosis Society; and International Association for the Study of Obesity. Circulation. 2009;120(16):1640-1645.

10. Kaur J. A comprehensive review on metabolic syndrome. Cardiol Res Pract. 2014;2014:943162.

11. Vestergaard P. Discrepancies in bone mineral density and fracture risk in patients with type 1 and type 2 diabetes--a meta-analysis. Osteoporos Int. 2007;18(4):427-444.

12. Kawai M, de Paula FJ, Rosen CJ. New insights into osteoporosis: the bone-fat connection. J Intern Med. 2012;272(4):317-329.

13. Iwaniec UT, Turner RT. Influence of body weight on bone mass, architecture and turnover. J Endocrinol. 2016;230(3):R115-R130.

14. Krakauer JC, McKenna MJ, Buderer NF, Rao DS, Whitehouse FW, Parfitt AM. Bone loss and bone turnover in diabetes. Diabetes. 1995;44(7):775-782.

15. Ho PC, Tsui YC, Feng X, Greaves DR, Wei LN. NF-kB-mediated degradation of the coactivator RIP140 regulates inflammatory responses and contributes to endotoxin tolerance. Nat Immunol. 2012;13(4):379-386.

16. Lin YW, Lee B, Liu PS, Wei LN. Receptor-interacting protein 140 orchestrates the dynamics of macrophage M1/M2 polarization. J Innate Immun. 2016;8(1):97-107.

17. Liu PS, Lin YW, Lee B, McCrady-Spitzer SK, Levine JA, Wei LN. Reducing RIP140 expression in macrophage alters ATM infiltration, facilitates white adipose tissue browning, and prevents high-fat diet-induced insulin resistance. Diabetes. 2014;63(12):4021-4031.

18. Teitelbaum SL. Bone resorption by osteoclasts. Science. 2000;289(5484):1504-1508

19. Yoshida $\mathrm{H}$, et al. The murine mutation osteopetrosis is in the coding region of the macrophage colony stimulating factor gene. Nature. 1990;345(6274):442-444.

20. Dougall WC, et al. RANK is essential for osteoclast and lymph node development. Genes Dev. 1999;13(18):2412-2424

21. Boyle WJ, Simonet WS, Lacey DL. Osteoclast differentiation and activation. Nature. 2003;423(6937):337-342.

22. Weivoda MM, et al. Osteoclast TGF- $\beta$ receptor signaling induces Wnt1 secretion and couples bone resorption to bone formation. J Bone Miner Res. 2016;31(1):76-85.

23. Cuetara BL, Crotti TN, O'Donoghue AJ, McHugh KP. Cloning and characterization of osteoclast precursors from the RAW264.7 cell line. In Vitro Cell Dev Biol Anim. 2006;42(7):182-188.

24. Huq MD, Gupta P, Tsai NP, Wei LN. Modulation of testicular receptor 4 activity by mitogen-activated protein kinase-mediated phosphorylation. Mol Cell Proteomics. 2006;5(11):2072-2082.

25. Lee YF, Young WJ, Burbach JP, Chang C. Negative feedback control of the retinoid-retinoic acid/retinoid X receptor pathway by the human TR4 orphan receptor, a member of the steroid receptor superfamily. J Biol Chem. 1998;273(22):13437-13443.

26. Zou W, Teitelbaum SL. Absence of Dap12 and the av $\beta 3$ integrin causes severe osteopetrosis. J Cell Biol. 2015;208(1):125-136.

27. Gingery A, Bradley EW, Pederson L, Ruan M, Horwood NJ, Oursler MJ. TGF-beta coordinately activates TAK1/MEK/AKT/ NFkB and SMAD pathways to promote osteoclast survival. Exp Cell Res. 2008;314(15):2725-2738.

28. Takayanagi H, et al. Induction and activation of the transcription factor NFATc1 (NFAT2) integrate RANKL signaling in termi- 
nal differentiation of osteoclasts. Dev Cell. 2002;3(6):889-901.

29. $\mathrm{Kim} \mathrm{HH}$, et al. Association of sustained ERK activity with integrin beta3 induction during receptor activator of nuclear factor kappaB ligand (RANKL)-directed osteoclast differentiation. Exp Cell Res. 2003;289(2):368-377

30. Canalis E. Clinical review 83: Mechanisms of glucocorticoid action in bone: implications to glucocorticoid-induced osteoporosis. J Clin Endocrinol Metab. 1996;81(10):3441-3447.

31. Iwaniec UT, Turner RT. Animal models of osteoporosis. In: Marcus R, Feldman D, Nelson DA, Rosen CJ, eds. Osteoporosis Amsterdam: Elsevier; 2008: 985-1110.

32. Collins LL, et al. Growth retardation and abnormal maternal behavior in mice lacking testicular orphan nuclear receptor 4. Proc Natl Acad Sci USA. 2004;101 (42):15058-15063.

33. Lin SJ, et al. Reduced osteoblast activity in the mice lacking TR4 nuclear receptor leads to osteoporosis. Reprod Biol Endocrinol. $2012 ; 10: 43$

34. Koga T, et al. Costimulatory signals mediated by the ITAM motif cooperate with RANKL for bone homeostasis. Nature. 2004;428(6984):758-763.

35. Zou W, et al. Syk, c-Src, the alphavbeta3 integrin, and ITAM immunoreceptors, in concert, regulate osteoclastic bone resorption. J Cell Biol. 2007;176(6):877-888.

36. Mendoza N, et al. Estrogen-related genes and postmenopausal osteoporosis risk. Climacteric. 2012;15(6):587-593.

37. Chen Y, Xia RG. Screening and functional microarray analysis of differentially expressed genes related to osteoporosis. Genet Mol Res. 2014;13(2):3228-3236.

38. Morón FJ, et al. Multilocus analysis of estrogen-related genes in Spanish postmenopausal women suggests an interactive role of ESR1, ESR2 and NRIP1 genes in the pathogenesis of osteoporosis. Bone. 2006;39(1):213-221.

39. Piao H, Chu X, Lv W, Zhao Y. Involvement of receptor-interacting protein 140 in estrogen-mediated osteoclasts differentiation, apoptosis, and bone resorption. J Physiol Sci. 2017;67(1):141-150.

40. Miotto PM, Frendo-Cumbo S, Sacco SM, Wright DC, Ward WE, Holloway GP. Combined high-fat-resveratrol diet and RIP140 knockout mice reveal a novel relationship between elevated bone mitochondrial content and compromised bone microarchitecture, bone mineral mass, and bone strength in the tibia. Mol Nutr Food Res. 2016;60(9):1994-2007.

41. Rao MK, Wilkinson MF. Tissue-specific and cell type-specific RNA interference in vivo. Nat Protoc. 2006;1(3):1494-1501.

42. Gough PJ, Gordon S, Greaves DR. The use of human CD68 transcriptional regulatory sequences to direct high-level expression of class A scavenger receptor in macrophages in vitro and in vivo. Immunology. 2001;103(3):351-361.

43. Philbrick KA, Turner RT, Branscum AJ, Wong CP, Iwaniec UT. Paradoxical effects of partial leptin deficiency on bone in growing female mice. Anat Rec (Hoboken). 2015;298(12):2018-2029.

44. Iwaniec UT, Wronski TJ, Turner RT. Histological analysis of bone. Methods Mol Biol. 2008;447:325-341.

45. van den Berg DL, et al. An Oct4-centered protein interaction network in embryonic stem cells. Cell Stem Cell. 2010;6(4):369-381.

46. Choi SH, et al. DUX4 recruits p300/CBP through its C-terminus and induces global H3K27 acetylation changes. Nucleic Acids Res. 2016;44(11):5161-5173. 